\title{
Effect of six degrees of freedom knee kinematics on ligament length and moment arm in an intact knee model
}

\author{
Neriman Ozada \\ Department of Mechanical Engineering, Eastern Mediterranean University, Gazimagusa, North \\ Cyprus, Mersin 10, Turkey \\ Tel.: +90 392630 1256; Fax: +90 392365 3715; E-mail: neriman.ozada@emu.edu.tr
}

Received 27 December 2014

Accepted 17 March 2015

\begin{abstract}
.
BACKGROUND: Biomechanics studies can help improve athletic performance. However, the biomechanics of knee joint ligament length changes and moment arms over six degrees of freedom (DOF) have yet to be established.

OBJECTIVE: To construct a knee model to investigate the length and moment arm changes of the anterior cruciate ligament (ACL), posterior cruciate ligament (PCL), medial collateral ligament (MCL), and lateral collateral ligament (LCL).

METHOD: Six DOF joint modeling and analysis were performed using specialized modeling software.

RESULTS: The length of all ligaments varied with tibiofemoral flexion angle, contributed to joint motion, and restrained the joint in different positions. The ACL, MCL, and LCL lengths decreased, the PCL increased, the posterior tibial translation increased, the MCL moment arm increased, and the LCL moment arm decreased between $0^{\circ}$ and $90^{\circ}$. The primary ligament restraints were the $\mathrm{PCL}\left(0^{\circ}\right.$ to $\left.30^{\circ}\right)$, $\mathrm{MCL}\left(30^{\circ}\right.$ to $\left.60^{\circ}\right)$, and $\mathrm{PCL}\left(60^{\circ}\right.$ to $\left.90^{\circ}\right)$.

CONCLUSION: The restraining function of each ligament during motion can be modeled based on changes in ligament lengths during tibial translations and rotations during flexion. Understanding the correlations between ligament lengths and moment arm changes over a wide range of motion will help improve our understanding of joint kinematics and may be useful for the diagnosis and treatment of sports injury.
\end{abstract}

Keywords: Biomechanics, anterior cruciate ligament, posterior cruciate ligament, medial collateral ligament, lateral collateral ligament

\section{Introduction}

The knee ligaments provide primary stability to the joint. The medial (MCL) and lateral collateral ligaments (LCL) are the primary knee stabilizers during valgus [112] and varus stresses [3], respectively, while the anterior (ACL) and posterior cruciate ligaments (PCL) are the primary stabilizers during anterior and posterior tibial translation, respectively [4|5]. Injury to the MCL may be associated with ACL injury, and injury to the LCL may be associated with PCL injury [6].

Several studies have evaluated the relative contributions of the knee ligaments to movement and function. These have included the contributions of the ACL and PCL to knee joint mobility [7]8], kinematic experiments on knee joints with ACL and MCL deficiencies [19|10], patterns of change of MCL and LCL length after total knee replacement [11], and changes in knee joint ligaments [12] and moment 
arms [13 14]. However, these empirical and theoretical studies have failed to determine the associations between the changes in knee ligament lengths and moment arms in all six degrees of freedom (DOF) of the knee joint. Understanding these interaction over all six DOF of knee joint kinematics would be useful for understanding mechanisms of sport injuries and developing diagnostic and treatment techniques for knee injuries.

Joint kinematics may be examined using different techniques. For instance, computer modeling of the knee joint and surrounding tissues has been useful [15]. Finite element analysis may help describe the stress-strain characteristics of the knee joint and surrounding tissues, but is unsuitable for assessing high DOF motion associated with ligament length changes and moment arms due to the high computational and time costs [16-19]. Multibody dynamics software studies may be used to simulate the effect of ligament deficiencies on the knee and provide faster joint analyses over wide ranges of motion [20]. However, any modeling solution should accurately represent the ligament and muscle lines of action and moment arms to realistically simulate joints, and inaccurate information about the soft tissue lines of action and moment arms may preclude complete kinematic joint simulation [21-24].

The purpose of this study was to model an intact knee joint over six DOF using recently developed software [25]. Changes in knee ligament lengths and moment arms were observed over six DOF of knee joint motion to evaluate the variations in these parameters.

\section{Materials and methods}

\subsection{Knee joint model construction}

The knee model was constructed using specialized joint modeling software (Musculoskeletal Joint Modeler; [25]). Briefly, this software was developed to investigate diarthrodial joints taking anatomic surface geometries into account to accurately model real anatomy. The surfaces are then interactively attached to tissue, tendons, and ligaments, and joint contact is described by automatically incorporating cartilage during analysis.

Here, anatomically accurate tibia and femur data were obtained by 3-dimensional (3D) laser-scanning (ZScanner $^{\mathrm{TM}} 800 \mathrm{Z}$ Corporation, Rock Hill, South Carolina, USA) of plastic sawbones (Skeleton Bert, Erler Zimmer, Lauf, Germany) and charting the surfaces in point clouds (where each point has its own coordinate). The origin and insertion points were selected via the computer screen (Geomagic, Geomagic Solutions, Morrisville, NC) [26] before being converted into 3D solid surfaces for import into the joint modeling software. Bones were described in terms of point clouds where each point has its own coordinate. The tibia and femur were modeled as rigid bodies. The ligaments (ACL, PCL, MCL, and LCL) were modeled as 3D linear elastic spring elements (spring-stiffness properties [k] for ACL and PCL, $100 \mathrm{~N} / \mathrm{mm}$; MCL and LCL, $65 \mathrm{~N} / \mathrm{mm}$ [20]), and articular cartilage was modeled as a single layer viscoelastic material between the tibia and femur (stiffness, $40 \mathrm{~N} / \mathrm{mm}$; damping, $400 \mathrm{Ns} / \mathrm{mm}$ [20]). A Cartesian coordinate system was established (translation: $+\mathrm{x}$, anterior; $-\mathrm{x}$, posterior; $+\mathrm{y}$, lateral; $-\mathrm{y}$, medial; $+\mathrm{z}$, superior; $-\mathrm{z}$, inferior), and polar coordinates were defined (rotation: $+\alpha$, varus [adduction]; $-\alpha$, valgus [abduction]; $+\beta$, flexion; $-\beta$, extension; $+\gamma$, internal rotation; $-\gamma$, external rotation) (Fig. 1). The six DOF kinematics included anterior-posterior, lateral-medial, and superior-inferior translations and varus-valgus, flexion-extension, and internal-external rotations. 


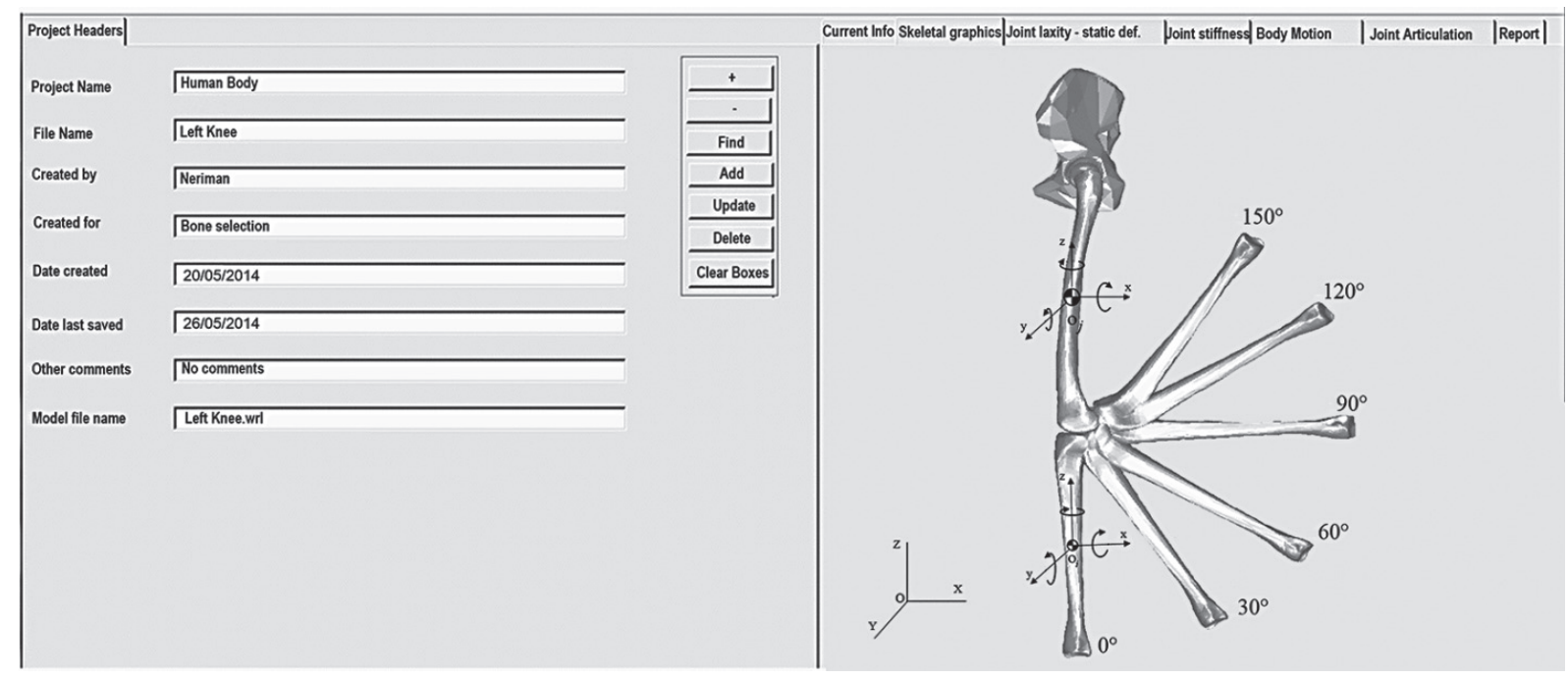

Fig. 1. Tibia and femur models with knee joint flexion angles from $0^{\circ}$ to $150^{\circ}$; local coordinate systems with the references $\mathrm{o}_{i}$ xyz for tibia and $\mathrm{o}_{j} \mathrm{xyz}$ for femur and global coordinate system with the reference OXYZ.

\subsection{Equations of motion}

The center of mass was calculated for each bone based on the surface geometry using a mathematical algorithm as previously described [27]. Calculation of the six DOF joint kinematics of the tibia and femur was performed by calculating the displacement of each ligament represented by a spring attached to the tibia and femur. The displacement of each ligament was described based on the translations, rotations, and position vectors of the attachment points. The displacement equation for each ligament was expressed as:

$$
\begin{aligned}
& \mathbf{d}_{t}=\mathbf{x}_{t}+\theta_{t} \times \mathbf{r}_{p t} \\
& \mathbf{d}_{f}=\mathbf{x}_{f}+\theta_{f} \times \mathbf{r}_{p f}
\end{aligned}
$$

where $p_{t}$ and $p_{f}$ were the ligament attachment points on the tibia and femur; $\mathbf{d}_{t}$ and $\mathbf{d}_{f}$ were the local displacements of the ligament attachment points; $\mathbf{r}_{p_{t}}$ and $\mathbf{r}_{p_{f}}$ were the position vectors of these attachment points; and $\mathbf{x}_{t}, \mathbf{x}_{f}, \theta_{t}$, and $\theta_{f}$ were the translations and rotations of the attached ligament.

Equations were assembled by considering the tibia and femur connected by spring $\mathbf{k}_{p}$ with the bodies rigid and all inertial properties assumed to be known. The motion of a linear spring element can be described by Hooke's law:

$$
\mathbf{F}=k \mathbf{x}
$$

where $\mathbf{F}$ is the ligament force, $k$ is the spring stiffness, and $\mathbf{x}$ is the displacement. The changes in the ligament length (scalar value of the displacement) were obtained in six DOF according to the displacement values of each ligament, where displacement is proportional to force to calculate the restraining functions of the ligaments.

The dynamic motion of a rigid body can be described by the translational and rotational motions of its center of mass; in this case, the center of mass of the tibia was $\mathrm{o}_{i}$ (Fig. 1), its translational motion was 


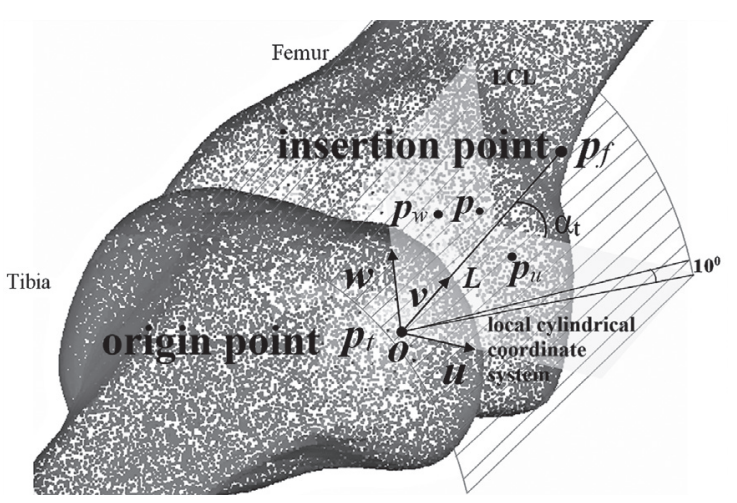

Fig. 2. Minimum surface point algorithm applied to the tibia and femur to draw ligament line of actions between the origin point $\mathrm{p}_{t}$ and the insertion point $\mathrm{p}_{f}$.

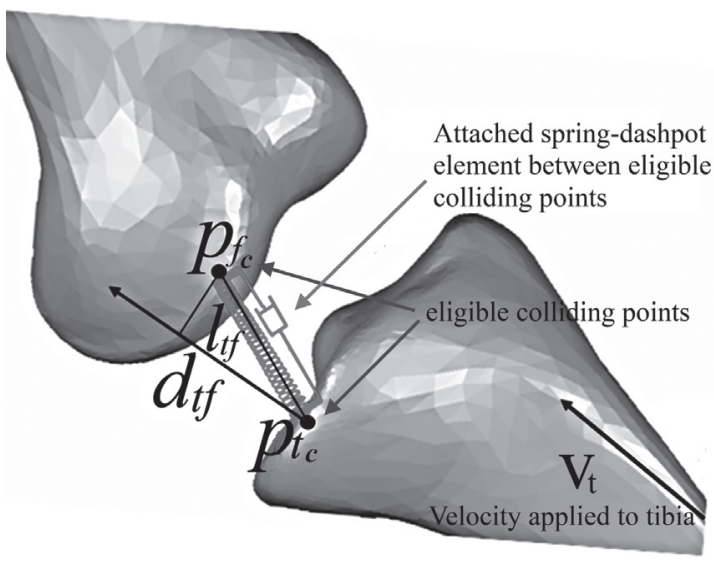

Fig. 3. Collision detection algorithm runs by applying the velocity $\mathrm{V}_{t}$ to the tibia. The spring-dashpot element was placed between the approaching surface points of tibia $\left(\mathrm{p}_{t}\right)$ and femur $\left(\mathrm{p}_{f}\right)$ to represent articular cartilage.

$\mathbf{x}_{t}$, and its rotational motion was $\theta_{t}$. Similarly, the translational motion of the femur was described by $\mathbf{x}_{f}$ and the rotational motion as $\theta_{f}$. In order to describe the spring force acting on each body, the location of the force due to the spring $\mathbf{k}_{p}$ was described using two position vectors $\mathbf{r}_{\mathbf{p}_{t}}$ and $\mathbf{r}_{\mathbf{p}_{f}}$ measured relative to local axes frames $\mathrm{o}_{i} \mathrm{x}_{i} \mathrm{y}_{i} \mathrm{z}_{i}$ and $\mathrm{o}_{j} \mathrm{x}_{j} \mathrm{y}_{j} \mathrm{z}_{j}$, respectively. Since the tibia and femur are connected by spring $\mathbf{k}_{p}$, the displacements of the spring end points are described in the axis frame of each body. The absolute displacement $\mathrm{p}_{t}$ is measured relative to the local axes $\mathrm{o}_{i} \mathrm{x}_{i} \mathrm{y}_{i} \mathbf{z}_{i}$ of the tibia as $\mathbf{d}_{t}$. The same description is valid for $\mathrm{p}_{f}$, which is measured relative to the local axes $\mathrm{o}_{j} \mathrm{x}_{j} \mathrm{y}_{j} \mathbf{z}_{j}$ of the femur as $\mathbf{d}_{f}$.

In this way, tibia and femur displacement based on ligament displacement generated six DOF joint kinematics without requiring consideration of kinematic joint constraints. The constructed Newton-Euler linear dynamic motion equations were represented in a global coordinate system as:

$$
\begin{gathered}
{\left[\begin{array}{cc}
\mathbf{m}_{t} & 0 \\
0 & \mathbf{J}_{t}
\end{array}\right]\left\{\begin{array}{l}
\ddot{\mathbf{x}}_{t} \\
\ddot{\boldsymbol{\theta}}_{t}
\end{array}\right\}-\left[\begin{array}{cc}
\mathbf{k}_{p} & \mathbf{k}_{p} \mathbf{R}_{p t} \\
\mathbf{R}_{p t}^{T} \mathbf{k}_{p} \mathbf{R}_{p t}^{T} \mathbf{k}_{p} \mathbf{R}_{p t}
\end{array}\right]\left\{\begin{array}{l}
\mathbf{x}_{t} \\
\boldsymbol{\theta}_{t}
\end{array}\right\}+\left[\begin{array}{ll}
\mathbf{k}_{p} & \mathbf{k}_{p} \mathbf{R}_{p f} \\
\mathbf{R}_{p t}^{T} \mathbf{k}_{p} \mathbf{R}_{p t}^{T} \mathbf{k}_{p} \mathbf{R}_{p f}
\end{array}\right]\left\{\begin{array}{l}
\mathbf{x}_{f} \\
\boldsymbol{\theta}_{f}
\end{array}\right\}=\left\{\begin{array}{l}
\mathbf{F}_{t} \\
\mathbf{M}_{t}
\end{array}\right\}} \\
{\left[\begin{array}{ll}
\mathbf{m}_{f} & 0 \\
0 & \mathbf{J}_{f}
\end{array}\right]\left\{\begin{array}{l}
\ddot{\mathbf{x}}_{f} \\
\ddot{\boldsymbol{\theta}}_{f}
\end{array}\right\}+\left[\begin{array}{ll}
\mathbf{k}_{p} & \mathbf{k}_{p} \mathbf{R}_{p t} \\
\mathbf{R}_{p t}^{T} \mathbf{k}_{p} \mathbf{R}_{p t}^{T} \mathbf{k}_{p} \mathbf{R}_{p t}
\end{array}\right]\left\{\begin{array}{l}
\mathbf{x}_{t} \\
\boldsymbol{\theta}_{t}
\end{array}\right\}-\left[\begin{array}{ll}
\mathbf{k}_{p} & \mathbf{k}_{p} \mathbf{R}_{p f} \\
\mathbf{R}_{p f}^{T} \mathbf{k}_{p} \mathbf{R}_{p f}^{T} \mathbf{k}_{p} \mathbf{R}_{p f}
\end{array}\right]\left\{\begin{array}{l}
\mathbf{x}_{f} \\
\boldsymbol{\theta}_{f}
\end{array}\right\}=\left\{\begin{array}{l}
\mathbf{F}_{f} \\
\mathbf{M}_{f}
\end{array}\right\}}
\end{gathered}
$$

where $\mathrm{m}$ was mass, $\mathrm{J}$ was the moment of inertia, $\mathrm{R}$ was the matrix form of the position vector $\mathbf{r}, \mathrm{k}_{p}$ was the spring stiffness, $\mathbf{x}$ was translation, $\boldsymbol{\theta}$ was rotation, $\mathbf{F}$ was the force vector acting along the line of action, and $\mathbf{M}$ was the moment vector. The transposed $\mathrm{R}$ matrix was denoted as $\mathbf{R}^{T}$.

\subsection{Line of action and moment arm calculations}

Estimates of lines of action and moment arms are important for accurate joint moment calculations. To generate ligament lines of action, we used a minimum surface point algorithm based on heuristic and surface geometry and points distributed along the bone surfaces as previously described [28]. To generate the line of action of a ligament, the origin point $\mathrm{p}_{t}$ and the insertion point $\mathrm{p}_{f}$ were selected interactively by the investigator (Fig. 2). The origin point $\mathrm{p}_{t}$ was selected, and a local cylindrical coordinate system was defined by the orthogonal vectors $\mathrm{u}, \mathrm{v}$, and $\mathrm{w}$. The insertion point $\mathrm{p}_{f}$ was selected, and a straight line $l$ was drawn between the origin and insertion points. The tibia and femur were then sliced into 
equal segments along vector $l$ with $10^{\circ}$ between each segment and the total number of surface points calculated for each segment; the segment containing the fewest number of surface points was selected for drawing the ligament line of action. Projection points were used to count the total number of points in each segment, and a projection angle was used to determine where a point belonged: point $\mathrm{p}$ was selected on the femur, and its projections on the $\mathrm{u}$ and $\mathrm{w}$ planes were determined as $\mathrm{p}_{u}$ and $\mathrm{p}_{w}$ (Fig. 2). The point $\mathrm{p}$ projection angle $\alpha_{i}$ was determined. The following equations were used to determine the projections and projection angles:

$$
\begin{aligned}
p_{u} & =\left(\mathbf{p}_{t}-\mathbf{p}_{f}\right) \cdot \mathbf{u} \\
p_{w} & =\left(\mathbf{p}_{t}-\mathbf{p}_{f}\right) \cdot \mathbf{w} \\
\alpha_{i} & =\tan ^{-1} \frac{p_{w}}{p_{u}}
\end{aligned}
$$

After drawing the ligament line of action, a convex hull algorithm was used to wrap the line around the bone surfaces between the attachment points.

Using the line of action and moment arm algorithms, the changes in ligament lengths and moment arms were measured. Firstly, the center of rotation $\mathbf{r}_{\text {cor }}$ at each knee joint flexion angle $\left(0^{\circ}, 30^{\circ}, 60^{\circ}\right.$, $90^{\circ}, 120^{\circ}, 150^{\circ}$ ) was determined. The length of a ligament was defined by $f_{a}$, which changed with respect to tibial angular rotation $\boldsymbol{w}$. The distance between the ligament line of action and the line parallel to tibial angular rotation $\boldsymbol{w}$ and passing through $\mathbf{r}_{\text {cor }}$ is casually known as the moment arm [25]. The moment arm of each ligament was defined by the relationship between the change in ligament length and tibial angular rotation:

$$
\text { moment arm }=\frac{\mathbf{f}_{a}}{|\mathbf{w}|}
$$

\subsection{Collision detection and response evaluation}

Collision detection and response calculations were performed as described previously [29]; briefly, a velocity $\boldsymbol{V}_{t}$ of the tibia toward the femur was applied and colliding point pairs on the tibial and femur surfaces recorded with the remaining surface points eliminated. Point $\boldsymbol{p}_{t c}$ on the tibia and point $\boldsymbol{p}_{f c}$ on the femur were potentially colliding point pairs observed by collision detection (Fig. 3). The collision detection algorithm determined whether these points were in the range of the collision trajectory by deriving constraint equations such as the shortest distance equation. The distance $\mathbf{d}_{t f}$ was the shortest distance between eligible colliding points $\boldsymbol{p}_{t c}$ and $\boldsymbol{p}_{f c}$ (Fig. 3). After finding eligible points, the collision response algorithm was applied with spring-dashpot elements attached to represent the articular cartilage between the eligible point pairs along length $\boldsymbol{l}_{t f}$.

All algorithms were implemented in Visual Basic (Microsoft, Redmond, WA) [15|25]; Visual Studio Online, 2013) and bone files were imported into the program in Virtual Reality Modeling Language file format.

\section{Results}

\subsection{Ligament length}

First, displacements of the ACL, PCL, MCL, and LCL were obtained using Eqs (1) and (2) to obtain the displacement of spring elements representing the displacement of ligaments. The ACL, PCL, MCL, 
Table 1

Changes in knee ligament length and kinematic parameters of the intact knee ${ }^{\mathrm{a}}$

\begin{tabular}{|c|c|c|c|c|c|c|}
\hline \multirow[t]{2}{*}{ Parameter } & \multicolumn{6}{|c|}{ Tibiofemoral flexion angle $\left(^{\circ}\right)$} \\
\hline & 0 & 30 & 60 & 90 & 120 & 150 \\
\hline \multicolumn{7}{|l|}{ Ligament length (mm) } \\
\hline $\mathrm{ACL}$ & 39.05 & 31.24 & 26.06 & 24.82 & 33.53 & 38.57 \\
\hline PCL & 32.41 & 33.59 & 35.75 & 36.95 & 36.01 & 34.11 \\
\hline LCL & 36.02 & 35.41 & 31.44 & 31.78 & 35.58 & 31.54 \\
\hline MCL & 71.30 & 71.47 & 73.78 & 66.21 & 79.92 & 68.43 \\
\hline \multicolumn{7}{|l|}{ Tibial translation (mm) } \\
\hline Anterior-posterior & -7.27 & -19.41 & -21.89 & -24.9 & -33.22 & -30.8 \\
\hline Lateral-medial & -1.19 & -3.63 & -2.33 & -8.67 & -5.92 & 0.08 \\
\hline Superior-inferior & 0.51 & 6.48 & 8.04 & 15.11 & 16.57 & 18.78 \\
\hline \multicolumn{7}{|l|}{ Rotation $\left(^{\circ}\right)$} \\
\hline Flexion-extension & 0 & 30 & 60 & 90 & 120 & 150 \\
\hline Varus-valgus & 0 & -2.63 & -4.39 & -7.15 & -8.18 & -3.51 \\
\hline Internal-external & 0 & 4.2 & 10.85 & 7.44 & 13.97 & 7.05 \\
\hline \multicolumn{7}{|l|}{ Moment arm (mm) } \\
\hline ACL & 7.94 & 2.64 & 2.09 & 6.55 & 6.7 & 3.58 \\
\hline PCL & 1.14 & 6.93 & 2.49 & 12.92 & 14.45 & 8.94 \\
\hline LCL & 38.2 & 32.6 & 32.9 & 23.1 & 22.5 & 25.4 \\
\hline MCL & 43.1 & 47.5 & 47 & 57.4 & 57 & 52.4 \\
\hline
\end{tabular}

${ }^{a}$ Positive and negative values defined for translation as $+\mathrm{x}$, anterior; $-\mathrm{x}$, posterior; $+\mathrm{y}$, lateral; $-\mathrm{y}$, medial; $+\mathrm{z}$, superior; $-\mathrm{z}$, inferior; and rotation as $+\alpha$, varus [adduction]; $-\alpha$, valgus [abduction]; $+\beta$, flexion; $-\beta$, extension; $+\gamma$, internal rotation; $-\gamma$, external rotation. Abbreviations: ACL, anterior cruciate ligament; LCL, lateral collateral ligament; MCL, medial collateral ligament; PCL, posterior cruciate ligament. The reference is the tibia COM, $\mathrm{o}_{i}(0,0,0)$.

and LCL lengths varied with tibiofemoral flexion angle over six DOF of the intact knee (Tables 1 and 2). Between $0^{\circ}$ and $90^{\circ}$ flexion, the tibia predominantly internally rotated, the ACL length decreased, and the PCL length increased. Between $0^{\circ}$ and $90^{\circ}$, the posterior tibial translation and MCL length increased. Increased medial tibial translation was mainly observed between $0^{\circ}$ to $90^{\circ}$ when the length of the MCL decreased. The ACL length increased with lateral tibial translations between $90^{\circ}$ and $150^{\circ}$ flexion, while at the same time the superior translation of the tibia increased markedly. Therefore, the ligament length changes cannot be distinguished based on the superior or inferior translations.

The PCL was longest, the MCL and ACL were shortest, and the tibia had the highest medial translation at $90^{\circ}$ flexion (Table 1). When the tibia was flexed from $0^{\circ}$ to $30^{\circ}$, it moved in valgus and internal rotation with posterior, superior, and latero-medial translation (Tables 1 and 2). During these movements, the ACL decreased by $20 \%$ and LCL decreased by $1.69 \%$ of their initial lengths, and the PCL increased by $3.64 \%$ and MCL increased by $0.23 \%$ (Table 2). Based on the ligament length increase, the PCL was the first and the MCL was the second restraint from $0^{\circ}$ to $30^{\circ}$ flexion.

When the tibia was flexed from $60^{\circ}$ to $90^{\circ}$, it moved in valgus and external rotation with superior, medial, and posterior translation; in this flexion range, the ACL decreased by $4.75 \%$, the MCL decreased by $10.26 \%$, the PCL increased by $3.35 \%$, and the LCL increased by $1.08 \%$ (Table 2). When the tibia was flexed from $90^{\circ}$ to $120^{\circ}$, it moved in valgus and internal rotation with posterior, lateral, and superior translation; the PCL decreased by $2.54 \%$ and the ACL (35.10\%), LCL (11.95\%), and MCL (20.70\%) increased. Between $120^{\circ}$ and $150^{\circ}$ flexion, the tibia moved in varus and external rotation with lateral, anterior, and superior translation (Tables 1 and 2); the PCL (5.27\%), LCL (11.35\%), and MCL (14.37\%) decreased and the ACL increased by $15.03 \%$. 
Table 2

Rate of knee joint ligament length and moment arm changes

\begin{tabular}{|c|c|c|c|c|c|c|}
\hline \multirow[t]{2}{*}{ Rate of parameter changes $(\%)$} & \multicolumn{6}{|c|}{ Tibiofemoral flexion angle $\left(^{\circ}\right)$} \\
\hline & $0-30$ & $30-60$ & $60-90$ & $90-120$ & $120-150$ & 150 \\
\hline \multicolumn{7}{|c|}{ Rate of ligament length changes $(\%)$} \\
\hline $\mathrm{ACL}$ & -20 & -16.58 & -4.75 & 35.10 & 15.03 & \\
\hline PCL & 3.64 & 6.43 & 3.35 & -2.54 & -5.27 & \\
\hline LCL & -1.69 & -11.21 & 1.08 & 11.95 & -11.35 & \\
\hline MCL & 0.23 & 3.23 & -10.26 & 20.7 & -14.37 & \\
\hline \multicolumn{7}{|l|}{ Rate of moment arm changes $(\%)$} \\
\hline ACL & -66.75 & -20.83 & 213.39 & 2.29 & -46.56 & \\
\hline PCL & 507.89 & -64.06 & 418.87 & 11.84 & -38.13 & \\
\hline LCL & -14.65 & 0.92 & -29.7 & -2.59 & 12.88 & \\
\hline MCL & 10.20 & -1.05 & 22.12 & -0.69 & -8.07 & \\
\hline
\end{tabular}

Table 3

Relationship between knee joint kinematics and ligament restraints

\begin{tabular}{|c|c|c|c|c|c|c|c|c|c|c|c|c|c|}
\hline \multirow{2}{*}{$\begin{array}{l}\text { Tibiofemoral } \\
\text { flexion } \\
\text { angle }\left(^{\circ}\right)\end{array}$} & \multicolumn{2}{|c|}{$\begin{array}{l}\text { Main secondary } \\
\text { joint rotation }\end{array}$} & \multicolumn{3}{|c|}{ Main joint translation } & \multicolumn{4}{|c|}{ Ligament restraints } & \multicolumn{4}{|c|}{$\begin{array}{c}\text { Maximum moment } \\
\text { arm change }\end{array}$} \\
\hline & $1^{\mathrm{st}}$ & $2^{\text {nd }}$ & $1^{\mathrm{st}}$ & $2^{\text {nd }}$ & $3^{r d}$ & $1^{\mathrm{st}}$ & $2^{\text {nd }}$ & $3^{r d}$ & $4^{t h}$ & $1^{\mathrm{st}}$ & $2^{\text {nd }}$ & $3^{r d}$ & $4^{\text {th }}$ \\
\hline 0 to 30 & Internal & Valgus & Posterior & Superior & Medial & PCL & MCL & $\mathrm{LCL}$ & ACL & PCL & $\mathrm{LCL}$ & $\mathrm{ACL}$ & MCL \\
\hline 30 to 60 & Internal & Valgus & Posterior & Superior & Lateral & MCL & PCL & LCL & $\mathrm{ACL}$ & PCL & ACL & MCL & $\mathrm{LCL}$ \\
\hline 60 to 90 & External & Valgus & Superior & Medial & Posterior & PCL & LCL & ACL & MCL & PCL & MCL & LCL & $\mathrm{ACL}$ \\
\hline 90 to 120 & Internal & Valgus & Posterior & Lateral & Superior & MCL & $\mathrm{ACL}$ & LCL & PCL & PCL & $\mathrm{LCL}$ & MCL & $\mathrm{ACL}$ \\
\hline 120 to 150 & External & Varus & Lateral & Anterior & Superior & ACL & PCL & LCL & MCL & PCL & MCL & ACL & LCL \\
\hline
\end{tabular}

Abbreviations: ACL, anterior cruciate ligament; LCL, lateral collateral ligament; MCL, medial collateral ligament; PCL, posterior cruciate ligament.

\subsection{Moment arm}

The ACL moment arm decreased by $66.77 \%$ between $0^{\circ}$ and $30^{\circ}$ and $20.83 \%$ between $30^{\circ}$ and $60^{\circ}$ flexion. The moment arm increased by $213.39 \%$ between $60^{\circ}$ and $120^{\circ}$ and by $2.29 \%$ between $90^{\circ}$ and $120^{\circ}$, and decreased by $46.56 \%$ between $120^{\circ}$ and $150^{\circ}$ (Table 2). The PCL moment arm increased between $0^{\circ}$ and $30^{\circ}$ and $60^{\circ}$ and $90^{\circ}$ flexion by $507.89 \%$ and $418.87 \%$, respectively. The PCL moment arm decreased between $30^{\circ}$ and $60^{\circ}$ and $120^{\circ}$ and $150^{\circ}$ by $64.06 \%$ and $38.13 \%$, respectively. The MCL moment arm increased between $0^{\circ}$ and $90^{\circ}$ flexion and decreased between $90^{\circ}$ and $150^{\circ}$ flexion.

The LCL moment arm decreased by $14.65 \%$ between $0^{\circ}$ and $30^{\circ}$ and slightly increased by $0.92 \%$ between $30^{\circ}$ and $60^{\circ}$. Between $60^{\circ}$ and $90^{\circ}$ flexion, the LCL moment arm decreased. The MCL and LCL moment arm changes were inversely proportional, while the MCL and PCL moment arm changes were proportional. The LCL moment arm changes were opposite to the PCL moment arm changes. Between $90^{\circ}$ and $120^{\circ}$, the MCL and PCL moment arms were maximum and the LCL minimum. While the MCL, ACL, and PCL moment arms peaked between $60^{\circ}$ and $150^{\circ}$, the LCL moment arm was lowest within this flexion angle range.

These findings suggest that each ligament has different moment arm changes between knee joint angles (Table 3 ) that directly affect the contribution of the ligaments to joint stability.

\section{Discussion}

In this study, we detail ligament length and moment arm correlations over six DOF of knee joint kinematics. These data could be used to help diagnose ligament injuries and treat ACL, PCL, MCL, and LCL 
ligaments during total knee arthroplasty and advocate the use of modeling based on multibody dynamics to predict knee joint behavior. Detailed characterization of ligament length changes over a wide range of knee motion may help improve tissue and joint diagnosis and treatment. For instance, in ligament reconstruction surgery, the ligament insertion point selected may affect the contribution of the ligament to joint torque during motion, and changing the direction of the ligament line of action may change its angular relationship to the moment arm and affect torque. A few previous studies have used experimental and mathematical models to evaluate ligament line of action and moment arm correlations, mostly with respect to changes in the knee muscle moment arms [13|14|30|31]. Our findings on ACL, PCL, LCL, and MCL moment arm trajectories are consistent with data from a previous cadaver study [14], and a previous theoretical study that predicted the trajectories of moment arm changes for the major knee ligaments were also similar [13].

The ACL length changes observed during knee motion corroborated previous findings that the lengths of the over-the-top single bundle (OT-SB), anatomical single bundle (ANA-SB) and anteromedial (AM) and posterolateral (PL) bundles of the ACL decrease between $0^{\circ}$ and $90^{\circ}$ and increase between $90^{\circ}$ and $120^{\circ}$ flexion [32]. In terms of the magnitudes of ACL length changes, these were found to be $10 \%$ different on average between the lengths of the AM bundle and the single-bundle ACL modeled in this study. The anterior, intermediate, and posterior fibers of the ACL have also been shown to change as a percentage of their reference lengths, similar to the results presented here [33].

The length changes of the anterolateral and posteromedial bundles of the PCL have previously been reported [34|35]. The reported anterolateral bundle length change pattern was similar to the singlebundle PCL length change reported here $(32.41 \mathrm{~mm}$ to $36.01 \mathrm{~mm}$ with increasing knee flexion angle from $0^{\circ}$ to $120^{\circ}$ ). In an in vivo study, Park et al. [36] demonstrated LCL length changes from $36.02 \mathrm{~mm}$ to $31.78 \mathrm{~mm}$ between $0^{\circ}$ and $90^{\circ}$ flexion, similar to our results.

By calculating the rates of ligament length changes over six DOF we could correlate the ligament restraints based on joint translation and rotation (Table 3). At each dominant translation and rotation, the restraining contribution of the ligaments was determined with the total increase in length. Our restraint data on the ACL are consistent with previous results showing that the ACL does not provide resistance to internal tibial rotation, especially at smaller flexion angles [37]. In the present study, when tibial internal rotation was the dominant rotation between $0^{\circ}$ and $60^{\circ}$ flexion, the ACL was the least restraining ligament with a $18.29 \%$ length decrease. With internal and valgus rotation of the tibia between $0^{\circ}$ and $30^{\circ}$ and $90^{\circ}$ and $120^{\circ}$ flexion, the MCL was the main ligament restraint with a $11.96 \%$ increase. However, the LCL became the second restraining ligament (1.08\% increase) when the tibia performed external rotation between $60^{\circ}$ and $90^{\circ}$ flexion. Therefore, internal-external rotation is dominant in intact knee motion and involves contributions from both the MCL and LCL.

This study has some limitations. A major limitation was the time cost associated with obtaining the surface data and manipulating it for import and analysis. For model simplicity, muscles and tendons were not included, even though they might be expected to contribute to stability and decrease the contribution of the ligaments to stability. This omission would not be expected to change the rate of the length changes of the ligaments at each flexion angle, but might change the position of the center of rotation and, therefore, the moment arm and length of the ligaments during articulation; the effects of muscles could be included in future studies. Furthermore, knee joint ligaments were only modeled as linear elastic single-bundle tissues; future studies could employ more sophisticated material models. 


\section{Conclusions}

Here, we model the restraining function of each knee joint ligament during motion based on the change in ligament lengths during tibial translations and rotations based on tibial flexion. Understanding the correlation between the ligament length and moment arm changes of each ligament during six DOF of knee motion will help improve our understanding of joint kinematics and may therefore be useful in the diagnosis and treatment of sports injuries.

\section{Acknowledgement}

This study was supported by the Institute of Graduate Studies at Eastern Mediterranean University, Gazimagusa, North Cyprus, Turkey.

\section{References}

[1] Matsumoto H, Suda Y, Otani T, Niki Y, Seedhom BB, Fujikawa K. Roles of the anterior cruciate ligament and the medial collateral ligament in preventing valgus instability. J Orthop Sci. 2001; 6: 28-32.

[2] Feeley BT, Muller MS, Allen AA, Granchi CC, Pearle AD. Isometry of medial collateral ligament reconstruction. Knee Surg Sports Traumatol Arthrosc. 2009; 17: 1078-1082. doi: 10.1007/s00167-009-0805-1.

[3] Grood ES, Noyes FR, Butler DL, Suntay WJ. Ligamentous and capsular restraints preventing straight medial and lateral laxity in intact human cadaver knees. J Bone Joint Surg Am. 1981; 63: 1257-1269.

[4] Dargel J, Gotter M, Mader K, Pennig D, Koebke J, Schmidt-Wiethoff R. Biomechanics of the anterior cruciate ligament and implications for surgical reconstruction. Strategies Trauma Limb Reconstr. 2007; 2: 1-12. doi: 10.1007/s11751-0070016-6.

[5] Amis AA, Bull AM, Gupte CM, Hijazi I, Race A, Robinson JR. Biomechanics of the PCL and related structures: posterolateral, posteromedial and meniscofemoral ligaments. Knee Surg Sports Traumatol Arthrosc. 2003; 11: 271-281.

[6] Hillard-Sembell D, Daniel DM, Stone ML, Dobson BE, Fithian DC. Combined injuries of the anterior cruciate and medial collateral ligaments of the knee. Effect of treatment on stability and function of the joint. J Bone Joint Surg Am. 1996; 78: 169-176.

[7] DeFrate LE, Gill TJ, Li G. In vivo function of the posterior cruciate ligament during weightbearing knee flexion. Am J Sports Med. 2004; 32: 1923-1928.

[8] Li G, Defrate LE, Rubash HE, Gill TJ. In vivo kinematics of the ACL during weight-bearing knee flexion. J Orthop Res. 2005; 23: 340-344.

[9] Lujan TJ, Dalton MS, Thompson BM, Ellis BJ, Weiss JA. Effect of ACL deficiency on MCL strains and joint kinematics. J Biomech Eng. 2007; 129: 386-392.

[10] Pressman, A., Johnson DH. A review of ski injuries resulting in combined injury to the anterior cruciate ligament and medial collateral ligaments. Arthroscopy. 2003; 19: 194-202.

[11] Ghosh KM, Merican AM, Iranpour F, Deehan DJ, Amis AA. Length-change patterns of the collateral ligaments after total knee arthroplasty. Knee Surg Sports Traumatol Arthrosc. 2012; 20: 1349-1356. doi: 10.1007/s00167-011-1824-2.

[12] Hosseini A, Qi W, Tsai T-Y, Liu Y, Rubash H, Li G. In vivo length change patterns of the medial and lateral collateral ligaments along the flexion path of the knee. Knee Surg Sports Traumatol Arthrosc. 2014; doi: 10.1007/s00167-0143306-9.

[13] Lu TW, O'Connor JJ. Lines of action and moment arms of the major force-bearing structures crossing the human knee joint: comparison between theory and experiment. J Anat, 189. 1996; 189: 575-585.

[14] Herzog W, Read LJ. Lines of action and moment arms of the major force-carrying structures crossing the human knee joint. J Anat. 1993; 182: 213-230.

[15] Shelburne KB, Torry MR, Pandy MG. Muscle, ligament, and joint-contact forces at the knee during walking. Med Sci Sports Exerc. 2005; 37: 1948-1956.

[16] Gardiner JC, Weiss JA. Subject-specific finite element analysis of the human medial collateral ligament during valgus knee loading. J Orthop Res. 2003; 21: 1098-1106.

[17] Phatak NS, Sun Q, Kim SE, Parker DL, Sanders RK, Veress AI, Ellis BJ, Weiss JA. Noninvasive determination of ligament strain with deformable image registration. Ann Biomed Eng. 2007; 35: 1175-1187. 
[18] Kiapour AM, Kaul V, Kiapour A, Quatman CE, Wordeman SC, Hewett TE, Demetropoulos CK, Goel VK. The effect of ligament modeling technique on knee joint kinematics: a finite element study. Applied Mathematics. 2013; 4(5A): 91-97. doi: 10.4236/am.2013.45A011.

[19] Zhang M, Fan Y. Computational Biomechanics of the Musculoskeletal System. 1st ed. CPC Press; 2014.

[20] Guess TM, Stylianou A. Simulation of anterior cruciate ligament deficiency in a musculoskeletal model with anatomical knees. Open Biomed Eng J. 2012; 6: 23-32. doi: 10.2174/1874230001206010023.

[21] Pandy MG. Moment arm of a muscle force. Exerc Sport Sci Rev. 1999; 27: 79-118.

[22] Gao F, Damsgaard M, Rasmussen J, Christensen ST. Computational method for muscle-path representation in musculoskeletal models. Biol Cybern. 2002; 87: 199-210.

[23] An KN, Takahashi K, Harrigan TP, Chao EY. Determination of muscle orientations and moment arms. J Biomech Eng. 1984; 106: 280-282.

[24] Adams CR, Baldwin MA, Laz PJ, Rullkoetter PJ, Langenderfer JE. Effects of rotator cuff tears on muscle moment arms: a computational study. J Biomech. 2007; 40: 3373-3380.

[25] Ozada N; 2008. A novel musculoskeletal joint modelling for orthopaedic applications [Ph.D. thesis]. Brunel University Research Archive Web site. http://v-scheiner.brunel.ac.uk/handle/2438/6556. Accessed June 1, 2014.

[26] Geomagic. Geomagic Studio v9. Geomagic Web site. http://www.geomagic.com. Accessed January 1, 2014.

[27] Mirtich B. Fast and accurate computation of polyhedral mass properties. J Graphics Tools. 1996; 1: 31-50. doi:10.1080/ 10867651.1996.10487458.

[28] Ozada N, Esat II. Development of tissue line of action and wrapping algorithms for musculoskeletal joint modelling [International Conference on Orthopaedic Biomechanics, Clinical Applications and Surgery, Abstract MJM-2]. J Biomech. 2010; 43(suppl 1): S16-S17.

[29] Ozada N, Esat II. Development of collision detection and collision response algorithms and modelling of human joint contact [International Conference on Orthopaedic Biomechanics, Clinical Applications and Surgery, Abstract MJM-3]. J Biomech. 2010; 43(suppl 1): S17-8.

[30] Buford WL, Ivey FM, Nakamura T, Patterson RM, Nguyen DK. Internal/external rotation moment arms of muscles at the knee: moment arms for the normal knee and the ACL-deficient knee. Knee. 2001; 8: 293-303.

[31] Fiorentino NM, Lin JS, Ridder KB, Guttman MA, McVeigh ER, Blemker SS. Rectus femoris knee muscle moment arms measured in vivo during dynamic motion with real-time magnetic resonance imaging. J Biomech Eng. 2013; 135: 044501. doi: 10.1115/1.4023523.

[32] Yoo YS, Jeong WS, Shetty NS, Ingham SJ, Smolinski P, Fu F. Changes in ACL length at different knee flexion angles: an in vivo biomechanical study. Knee Surg Sports Traumatol Arthrosc. 2010; 18: 292-297. doi: 10.1007/s00167-0090932-8.

[33] Imran A. Anterior cruciate ligament fibres - effects of tibial translation during flexion at the knee. Proceedings of the World Congress on Engineering. London, UK; 2012: 1-4.

[34] Bowman KF, Sekiya JK. Anatomy and biomechanics of the posterior cruciate ligament and other ligaments of the knee. Operative Techniques in Sports Medicine. 2009; 17: 126-134. doi: 10.1053/j.otsm.2009.07.001.

[35] Ahmad CS, Cohen ZA, Levine WN, Gardner TR, Ateshian GA, Mow VC. Codominance of the individual posterior cruciate ligament bundles. An analysis of bundle lengths and orientation. Am J Sports Med. 2003; 31: 221-225.

[36] Park SE, DeFrate LE, Suggs JF, Gill TJ, Rubash HE, Li G. The change in length of the medial and lateral collateral ligaments during in vivo knee flexion. Knee. 2005; 12: 377-382.

[37] Wünschel M, Müller O, Lo J, Obloh C, Wülker N. The anterior cruciate ligament provides resistance to externally applied anterior tibial force but not to internal rotational torque during simulated weight-bearing flexion. Arthroscopy. 2010; 26: 1520-1527. doi: 10.1016/j.arthro.2010.04.069. 